\title{
MicroRNA-424-5p inhibits the proliferation, migration, and invasion of nasopharyngeal carcinoma cells by decreasing AKT3 expression
}

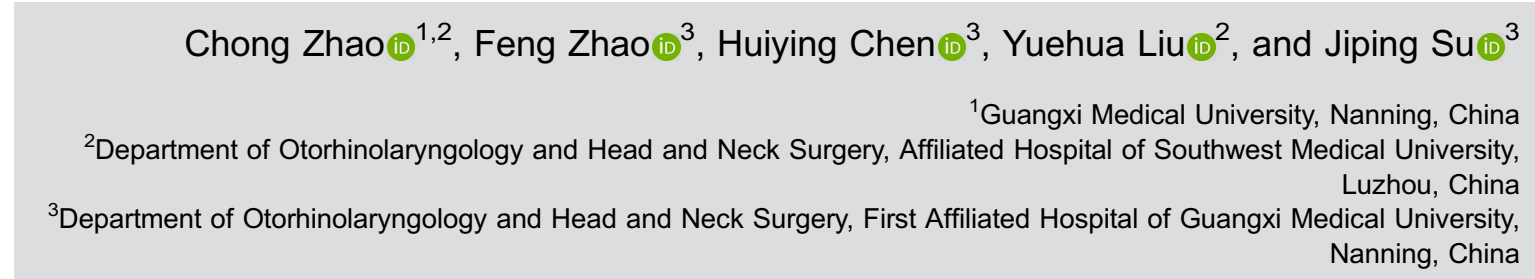

\begin{abstract}
This study examined the expression and potential mechanism of microRNA (miRNA)-424-5p in nasopharyngeal carcinoma (NPC). NPC tissues were collected from 40 patients who were enrolled in the study, and skin samples were collected from 26 healthy subjects during plastic surgery as controls. We performed various in vitro assays using miR-424-5p to examine its function in primary NPC-1 cells. Bioinformatics was employed to analyze potential target genes and signaling pathways of miR424-5p. We found that miR-424-5p expression in NPC tissues is downregulated and negatively correlated with lymph node metastasis and clinical staging. Expression of miR-424-5p in NPC cells was also downregulated, and transfection with miR-424$5 p$ mimics inhibited proliferation, migration, and invasion of NPC-1 cells. Bioinformatics identified the AKT3 gene as a potential target of miR-424-5p and dual luciferase assays confirmed this finding. Upregulation of AKT3 expression rescued the inhibitory effect of miR-424-5p on the proliferation, migration, and invasion. Our results suggest that miR-424-5p inhibited the proliferation, migration, and invasion of NPC cells by decreasing AKT3 expression.
\end{abstract}

Key words: Nasopharyngeal carcinoma; microRNA-424-5p; AKT3

\section{Introduction}

Nasopharyngeal carcinoma (NPC) is a type of head and neck cancer with a high incidence in southern China and Southeast Asia (1). Similar to most tumors, the occurrence of NPC is closely related to genetic factors, infection by virus such as Epstein-Barr virus, and environmental factors (2). Low-differentiation squamous cell carcinoma is the common clinical type of NPC, while highly differentiated squamous cell carcinoma, adenocarcinoma, and alveolar cell carcinoma are rare types of NPC (3). Because of the special anatomical position of NPC, radiation therapy is often used by clinicians as the first choice for treatment (4). In recent years, the progress of radiotherapy and chemotherapy and the development of new tumor therapies such as immunotherapy and precision medicine have greatly improved the treatment options for NPC. However, a considerable number of NPC patients still show drug resistance, recurrence, and tumor metastasis, which seriously restrict the effects of clinical treatment for the disease $(5,6)$. Studies have shown that the recurrence and metastasis of NPC are the main causes of death (7).
Invasion and metastasis of NPC is a multi-gene, multistage, and multi-step complex process that involves many factors (7). In addition, the molecular mechanism of NPC remains to be completely elucidated (8). Therefore, clarifying the molecular mechanism of invasion and metastasis of NPC to search for molecular targets that are related to the malignant biological behaviors of NPC is critical for improving the clinical treatment of NPC.

MicroRNA (miRNA) is a class of non-encoding small RNA molecules that are 18-25 nucleotides in length. miRNAs interact with the $3^{\prime}$-untranslated region (UTR) of target mRNAs to form transcriptional silencing complexes, organize the translation of proteins, and regulate the expression of genes $(9,10)$. Several studies have demonstrated various roles for miRNAs in cancer, and miRNAs can function either as oncogenes or tumor suppressor genes to regulate the occurrence and development of tumors (11). Furthermore, aberrant expression of these oncogenic and tumor suppressive miRNA molecules has been identified in multiple cancers, leading to changes in

Correspondence: Jiping Su: <jpsu2020@126.com> 
cell proliferation, invasion, and metastasis $(14,15)$. For example, miR-16 inhibits the proliferation and metastasis of hepatocellular carcinoma (12), and miR-25 promotes the proliferation and invasion of gastric cancer by regulating ERBB2 (13). Therefore, examining miRNA molecules that are abnormally expressed in tumors and participate in the invasion and metastasis of the tumors is of great clinical importance.

The has-miR-424-5p gene is a newly discovered miRNA located on the human Xq26.3 chromosome. miR424-5p targets different genes in different tissues, suggesting its diversified functions (16). Abnormal expression of miR-424-5p has been detected in many tumor tissues. For example, miR-424-5p is upregulated in esophageal squamous cell carcinoma, colorectal cancer, and nonsmall cell lung cancer, but its expression in cervical cancer is downregulated, suggesting that miR-424-5p is differentially expressed in different tumors $(17,18)$. Currently, the expression and function of miR-424-5p in NPC are still unclear. In the present study, we evaluated the expression and potential function of miR-424-5p in NPC.

\section{Material and Methods}

\section{Patients}

Forty NPC patients who received treatments at our hospital between January 2015 and January 2016 were enrolled in the present study. The patient group included 21 males and 19 females (mean, 48.5 years; range 31-68 years). None of the patients had a history of other types of malignant tumors, chemotherapy, or radiotherapy. Among all patients, 27 had cervical lymph node metastasis and 13 had no cervical lymph node metastasis. Based on TNM staging and grading standards by the Union for International Cancer Control in 2003, 11 cases were at stage I, 9 were at stage II, 15 were at stage III, and 5 were at stage IV. According to pathological classification, 35 cases had poorly differentiated squamous cell carcinoma and 5 had highly differentiated squamous cell carcinoma. NPC tissues were collected from the 40 patients as the experimental group and skin samples collected from 26 healthy subjects during plastic surgery were used as controls. All procedures were approved by the Ethics Committee of Guangxi Medical University. Written informed consent was obtained from all patients or their families.

\section{Primary cell culture}

Excised human NPC tissues were washed with phosphate-buffered saline and soaked in penicillin $\left(5 \times 10^{5} \mathrm{U} /\right.$ L) and streptomycin (100 mg/L) for $20 \mathrm{~min}$. The tissues were cut into $1-\mathrm{mm}^{3}$ pieces and digested with $30 \mathrm{~mL}$ collagenase I $\left(2 \times 10^{5} \mathrm{U} / \mathrm{L}\right)$ at $37^{\circ} \mathrm{C}$ for $2 \mathrm{~h}$. After filtration with a 100-mesh cell sieve, the filtrate was centrifuged at $1000 \mathrm{~g}$ for $10 \mathrm{~min}$ at room temperature before discarding the supernatant. The pellet was washed twice with phosphate-buffered saline and resuspended with RPMI-1640 medium supplemented with $10 \%$ fetal bovine serum in culture flasks with a bottom area of $25 \mathrm{~cm}^{2}$. Immortalized nasopharyngeal epithelial NP69 cells (Cell Bank, Chinese Academy of Sciences, China) were cultured in RPMI-1640 medium supplemented with $10 \%$ fetal bovine serum, $100 \mathrm{IU} / \mathrm{mL}$ penicillin, and $100 \mathrm{IU} / \mathrm{mL}$ streptomycin at $37^{\circ} \mathrm{C}$, $5 \% \mathrm{CO}_{2}$, and $70 \%$ humidity. The cells were passaged every 3 days.

\section{Transfection of miRNA mimics}

One day before transfection, NPC-1 cells $\left(2 \times 10^{5}\right)$ in logarithmic growth were seeded into 24-well plates containing antibiotic-free RPMI-1640 medium with $10 \%$ fetal bovine serum for culture until cells reached $70 \%$ confluency. In one vial, $1.5 \mu \mathrm{L}$ miR-negative control $(20 \mathrm{pmol} / \mu \mathrm{L}$; miRNC group) or miR-424-5p mimics (20 pmol/ $\mu \mathrm{L}$; miR-424-5p mimics group) (Hanbio Biotechnology Co., Ltd., China) were mixed with $50 \mu \mathrm{L}$ Opti Mem medium (Thermo Fisher Scientific, USA). In a second vial, $1 \mu \mathrm{L}$ Lipofectamine 2000 (Thermo Fisher Scientific) was mixed with $50 \mu \mathrm{L}$ Opti Mem medium. After being allowed to stand for $5 \mathrm{~min}$, the two vials were combined and held at room temperature for $20 \mathrm{~min}$. The mixtures were added onto the appropriate cells. Six hours later, the medium was replaced with RPMI1640 medium containing $10 \%$ fetal bovine serum. After culture for $48 \mathrm{~h}$, the cells were collected for further assays.

\section{Quantitative real-time polymerase chain reaction (qRT-PCR)}

NPC tissues (100 mg) were ground into powder in liquid nitrogen and lysed using $1 \mathrm{~mL}$ TRIzol reagent (Thermo Fisher Scientific) following the manufacturer's manual. Total RNA was extracted using phenol chloroform. The concentration and quality of RNA was measured using ultraviolet spectrophotometry (Nanodrop ND2000, Thermo Scientific). cDNA was obtained by reverse transcription from $1 \mu \mathrm{g}$ RNA and samples were stored at $-20^{\circ} \mathrm{C}$. Reverse transcription of miRNA was carried out using the miScript II RT Kit (Qiagen, Germany) following the manufacturer's manual.

The expression of miR-424-5p was determined with the miScript SYBR Green PCR Kit (Qiagen), using U6 as an internal reference. The forward primer sequence of miR-424-5p primer was 5'-CAGCAGCAATTCATGTTTT GAA-3' and its reverse primer sequence was universal and not provided by the supplier. The sequences of the U6 primers were forward, 5'-CTCGCTTCGGCAGCACA-3' and reverse 5'-AACGCTTCACGAATTTGCGT-3'. The reaction system $(20 \mu \mathrm{L})$ contained $10 \mu \mathrm{L}$ qRT-PCR-Mix, $0.5 \mu \mathrm{L}$ forward primer, $0.5 \mu \mathrm{L}$ reverse primer, $2 \mu \mathrm{L} \mathrm{cDNA}$, and $7 \mu \mathrm{L} \mathrm{ddH}_{2} \mathrm{O}$. The reaction protocol was as follows: initial denaturation at $95^{\circ} \mathrm{C}$ for $10 \mathrm{~min}$, followed by 40 cycles of denaturation at $95^{\circ} \mathrm{C}$ for $1 \mathrm{~min}$, and annealing at $60^{\circ} \mathrm{C}$ for $30 \mathrm{~s}$ (iQ5; Bio-Rad, USA). The $2^{-\Delta \Delta \mathrm{Ct}}$ method (19) was used to calculate the relative expression of miR424-5p against U6. Each sample was tested in triplicate. 
Nude mouse model of subcutaneous tumorigenesis

Five nude mice were selected randomly and received subcutaneous inoculation with primary NPC cells $\left(1 \times 10^{6}\right)$. The mice in the control group were injected with an equal amount of saline. The next day, the injection sites in nude mice were observed to check the degree of absorption. The tumor size was observed and measured every day.

Construction of nude mouse model of lung metastasis

Eight male nude mice were randomly divided into two groups, and NPC-1 $\left(5 \times 10^{5}\right)$ and NPC-2 $\left(5 \times 10^{5}\right)$ cells were injected into each nude mouse via tail vein. Nude mice were observed daily and executed 14 days later. The lung tissue was dissected, and the lung cancer nodules were observed. The tissues were stained with hematoxylin and eosin.

\section{CCK-8 assay}

NPC- 1 cells were seeded at a density of $2000 /$ well in 96-well plates. At 0, 24, 48, and $72 \mathrm{~h}$ later, $20 \mu \mathrm{L}$ CCK-8 reagent ( $5 \mathrm{~g} / \mathrm{L}$; Beyotime, China) was added to the cells. After incubation at $37^{\circ} \mathrm{C}$ for $2 \mathrm{~h}$, the absorbance of each well was measured at $490 \mathrm{~nm}$ and cell proliferation curves were plotted. Each group was tested in three replicate wells and the values were averaged.

\section{Transwell assay}

Cell migration and invasion were determined using 24well transwell plates (Corning Inc., USA) with 8- $\mu \mathrm{m}$-pore polycarbonate membranes. Matrigel (BD Biosciences, USA) was thawed at $4^{\circ} \mathrm{C}$ overnight and diluted with serum-free RPMI-1640 medium (dilution 1:2). The mixture (50 $\mu \mathrm{L}$ ) was evenly smeared into the upper chamber (Merck Millipore, USA) and the chambers were incubated at $37^{\circ} \mathrm{C}$ for $1 \mathrm{~h}$. After solidification, $1 \times 10^{5}$ cells from each group were seeded into the upper chamber containing $200 \mu \mathrm{L}$ serumfree RPMI-1640 medium. Next, $500 \mu \mathrm{L}$ RPMI-1640 medium supplemented with $10 \%$ fetal bovine serum was added into the lower chamber. After $24 \mathrm{~h}$, the chamber was removed and the cells in the upper chamber were wiped off. After fixation with $4 \%$ formaldehyde for $10 \mathrm{~min}$, the membrane was stained using the Giemsa method for microscopic observation. The number of migrated cells was calculated and averaged in five random fields $(200 \times)$. All procedures were carried out on ice with pipetting tips precooled at $4^{\circ} \mathrm{C}$.

\section{Bioinformatics}

We searched http://www.targetscan.orgwebsite for "miR-424-5p" to identify potential target genes. These potential target genes were submitted to the David database (https://david.ncifcrf.gov/), and the keyword "human" was selected. The potential downstream signaling pathways were analyzed.

\section{Western blotting}

NPC-1 cells were trypsinized and collected. Cells $\left(1 \times 10^{6}\right)$ in each group were lysed with precooled radio-immunoprecipitation assay (RIPA) lysis buffer (600 $\mu \mathrm{L} ; 50 \mathrm{mM}$ Tris-base, $1 \mathrm{mM}$ EDTA, $150 \mathrm{mM} \mathrm{NaCl}, 0.1 \%$ sodium dodecyl sulfate, $1 \%$ TritonX-100, $1 \%$ sodium deoxycholate; Beyotime Institute of Biotechnology, China) for $30 \mathrm{~min}$ on ice. The sample was centrifuged at $12,000 \mathrm{~g}$ and $4^{\circ} \mathrm{C}$ for $10 \mathrm{~min}$. Protein concentration of the supernatant was determined by the bicinchoninic acid (BCA) protein concentration determination kit (RTP7102, RealTimes (Beijing) Biotechnology Co., Ltd., China). The samples were then mixed with $5 \times$ sodium dodecyl sulfate loading buffer before denaturation in a boiling water bath for $10 \mathrm{~min}$. Samples $(10 \mu \mathrm{L})$ were then subjected to separation by $10 \%$ sodium dodecyl sulfate-polyacrylamide gel electrophoresis at $100 \mathrm{~V}$. The resolved proteins were transferred to polyvinylidene difluoride membranes on ice (250 mA, $1 \mathrm{~h}$ ) and blocked with $50 \mathrm{~g} / \mathrm{L}$ skimmed milk at room temperature for $1 \mathrm{~h}$. Membranes were incubated with rabbit anti-human AKT3 polyclonal primary antibody (1:1000; ab152157; Abcam, UK) or mouse anti-human GAPDH monoclonal primary antibody (1:4000; Beyotime) at $4^{\circ} \mathrm{C}$ overnight. After extensive washing with phosphate-buffered saline with Tween 20 five times for 5 min each, the membranes were incubated with goat anti-rabbit or goat anti-mouse horseradish peroxidase-conjugated secondary antibodies $(1: 4,000$; Abcam) for $1 \mathrm{~h}$ at room temperature before washing with phosphate-buffered saline with Tween 20 five times for 5 min each. The membrane was developed with an enhanced chemiluminescence detection kit (Beyotime) for imaging. Image Lab v3.0 software (Bio-Rad, USA) was used to acquire and analyze imaging signals. The relative content of the target protein was normalized to GAPDH.

\section{Dual luciferase reporter assay}

Based on the bioinformatics results, wild-type (WT) and mutant seed regions of miR-424-5p in the $3^{\prime}-$ UTR of AKT3 gene were chemically synthesized in vitro. Spe-1 and HindIII restriction sites were included on either end and the fragment was then cloned into pMIR-REPORT luciferase reporter plasmids. Plasmids $(0.5 \mu \mathrm{g})$ with WT or mutant $3^{\prime}$-UTR sequences were co-transfected with agomiRnegative control (NC) or agomiR-424-5p (100 nM; Sangon Biotech, China) into 293T cells. After cultivation for $24 \mathrm{~h}$, the cells were lysed and processed using the dual luciferase reporter assay kit (Promega, USA) according to the manufacturer's manual. Fluorescence intensity was measured using GloMax 20/20 luminometer (Promega). The fluorescence values of each group of cells were measured using renilla fluorescence activity as an internal reference.

\section{Statistical analysis}

The results were analyzed using SPSS 20.0 statistical software (IBM, USA). Data are reported as means \pm SD. Data were tested for normality. Multigroup measurement data were analyzed using one-way ANOVA. In case of homogeneity of variance, the least significant difference and Student-Newman-Keuls methods were used; in case 
of heterogeneity of variance, Tamhane's T2 or Dunnett's T3 method was used. Comparison between two groups was carried out using Student's $t$-test. $\mathrm{P}<0.05$ indicated statistically significant differences.

\section{Results}

Expression of miR-424-5p was reduced in NPC tissues and correlated with the occurrence and development of NPC

The relative expression of miR-424-5p in NPC tissues $(0.25 \pm 0.05)$ was significantly lower than that in the control group $(P<0.05)$ (Figure $1 \mathrm{~A})$. In addition, the relative expression of miR-424-5p in NPC tissues from patients with lymph node metastasis $(0.48 \pm 0.05 ; \mathrm{N} 1$ group) was significantly lower than that from patients without lymph node metastasis (N0 group; $\mathrm{P}<0.05$ ) (Figure 1B). The relative expression of miR-424-5p in NPC tissues at TNM stage II or at stages III and IV was significantly lower than that in NPC tissues at stage I $(P<0.05)$ (Figure $1 C)$. We also observed that miR-424-5p expression in NPC cell lines was significantly lower than that in normal nasopharyngeal epithelial NP69 cells ( $P<0.05)$ (Figure 1D). These results suggested that the expression of miR-424-5p was reduced in NPC tissues and correlated with the occurrence and development of NPC.

\section{Isolation of primary NPC-1 and NPC-2 cells}

The morphologies of NPC-1 and NPC-2 cells are shown in Figure 2A. We used these cell lines for subcutaneous inoculation in mice and observed tumors at injection sites on day 7 (Figure 2B). Lung metastasis tests showed that the inoculated NPC-1 and NPC-2 cells metastasized to the lungs (Figure 2C). qRT-PCR showed
A

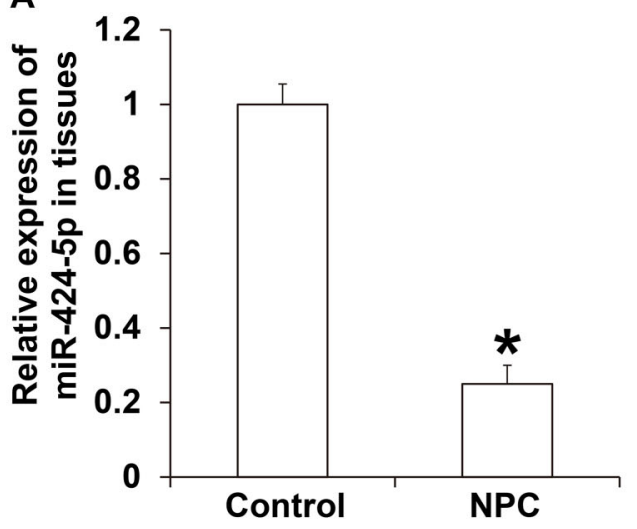

C

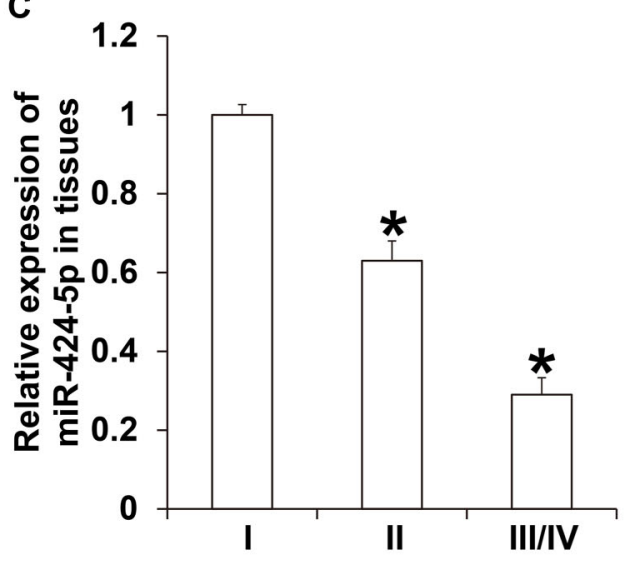

B
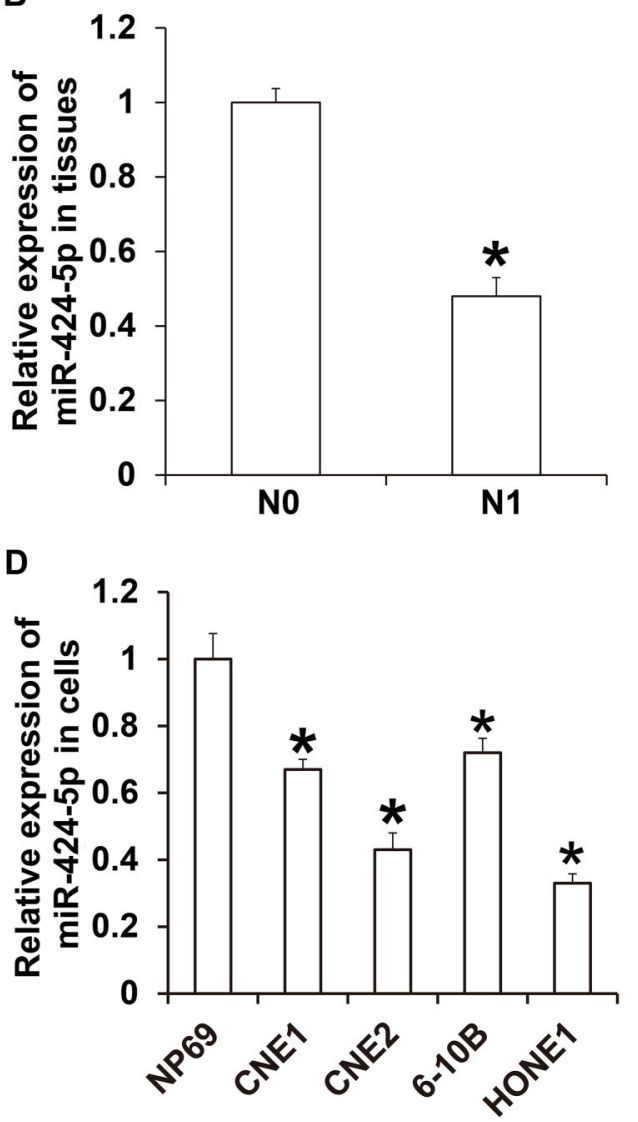

Figure 1. A, Expression of miR-424-5p in nasopharyngeal carcinoma (NPC) tissues and control tissues. ${ }^{*} \mathrm{P}<0.05$ compared with control group. B, Expression of miR-424-5p in NPC tissues from patients with (N1) or without (N0) lymph node metastasis. * $P<0.05$ compared with the N0 group. C, Expression of miR-424-5p in NPC tissues from patients at TNM stages I, II, or III/IV. ${ }^{*} P<0.05$ compared with stage I. D, Expression of miR-424-5p in NPC cells and normal nasopharyngeal epithelial NP69 cell lines. Data are reported as means $\pm S D$. ${ }^{*} P<0.05$ compared with NP69 cells ( $t$-test or ANOVA). 
A

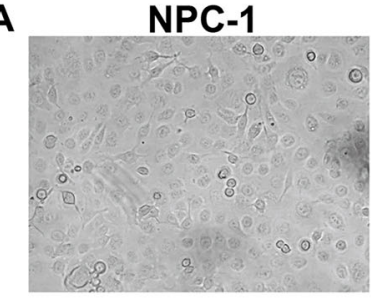

B

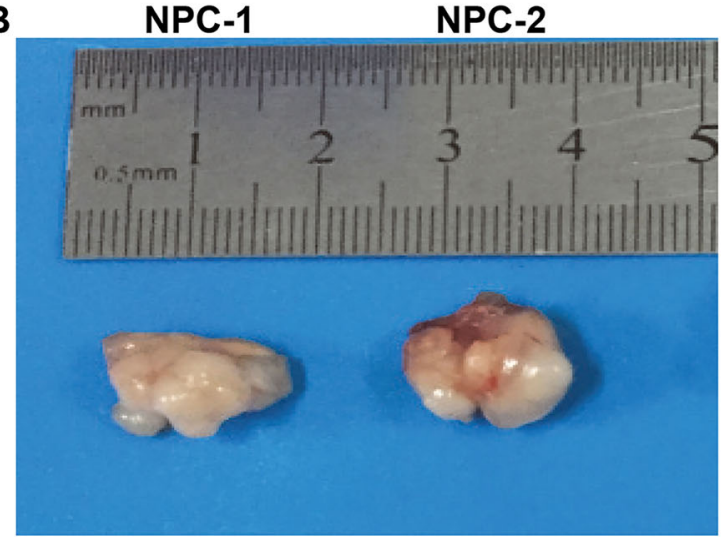

C

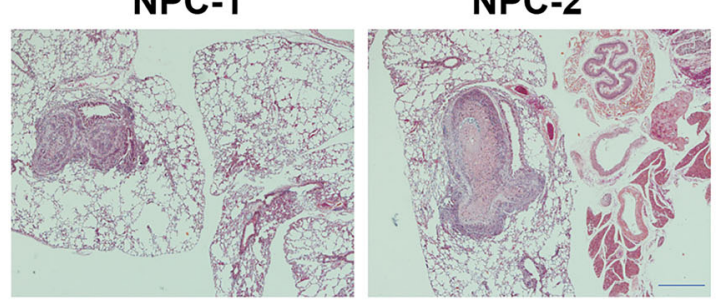

D

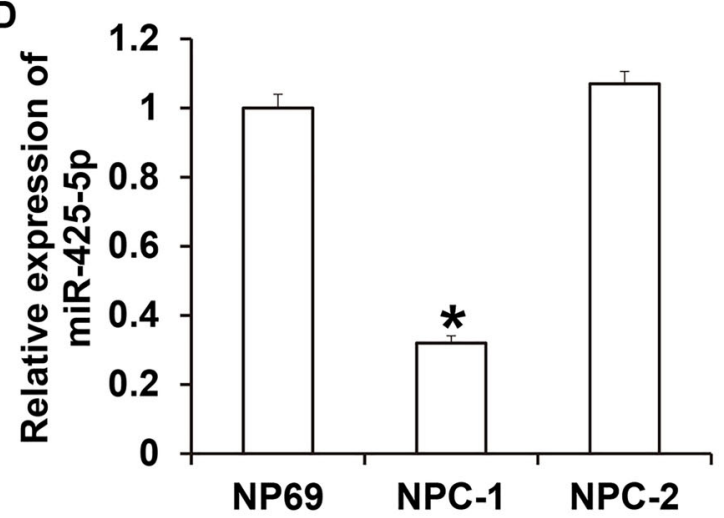

Figure 2. Identification of primary nasopharyngeal carcinoma (NPC) cells. A, Light microscopy of primary NPC-1 and NPC-2 cells (scale bar: $1 \mu \mathrm{m}$ ). B, Tumorigenicity of NPC-1 and NPC-2 in nude mice. C, Lung metastasis of NPC-1 and NPC-2 cells (scale bar: $100 \mu \mathrm{m}$ ). D, Expression of miR-425-5p in NPC-1 and NPC-2 cells. Data are reported as means $\pm S D$. ${ }^{*} P<0.05$ compared with NP69 cells (ANOVA).
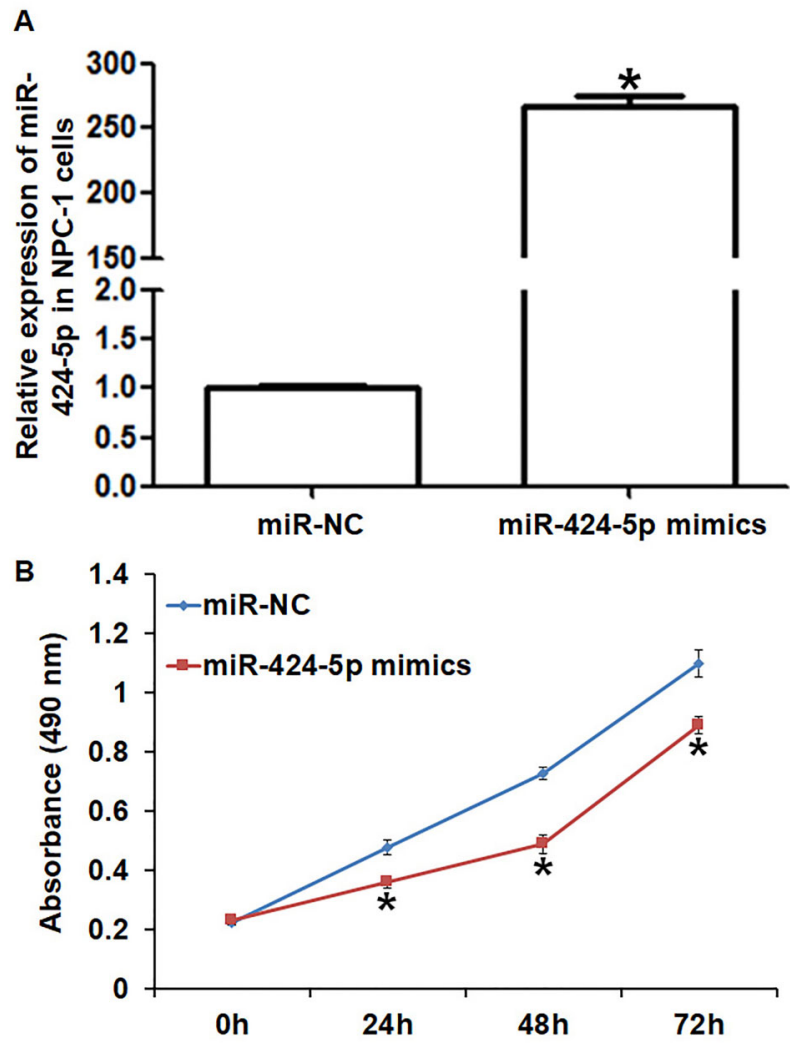

Figure 3. Effect of miR-424-5p on the proliferation of nasopharyngeal carcinoma (NPC)-1 cells. A, Expression of miR-424$5 p$ in NPC-1 cells after transfection with miR-NC (negative control) or miR-424-5p mimics. B, Proliferation of NPC-1 cells after transfection with miR-NC or miR-424-5p mimics. CCK-8 assay was used to detect cell proliferation. Data are reported as means $\pm S D$. ${ }^{*} \mathrm{P}<0.05$ compared with the miR-NC group (t-test).

that the expression of miR-425-5p in NPC-1 cells was significantly lower than that in NP69 cells $(P<0.05)$ (Figure 2D). The results suggested that primary NPC-1 and NPC-2 cells were successfully isolated.

\section{Overexpression of $\mathrm{miR}-424-5 p$ inhibited proliferation of NPC-1 cells in vitro}

miR-424-5p expression in the miR-424-5p mimics group was significantly higher than that in the miR-NC group $(P<0.05)$ (Figure $3 A)$. CCK-8 assay showed the miR-424-5p mimics group had significantly decreased growth at 24, 48, and $72 \mathrm{~h}$ compared with the miR-NC group ( $P<0.05$ at all points) (Figure $3 B$ ). These results indicated that overexpression of miR-424-5p inhibited the proliferation of NPC-1 cells in vitro. 

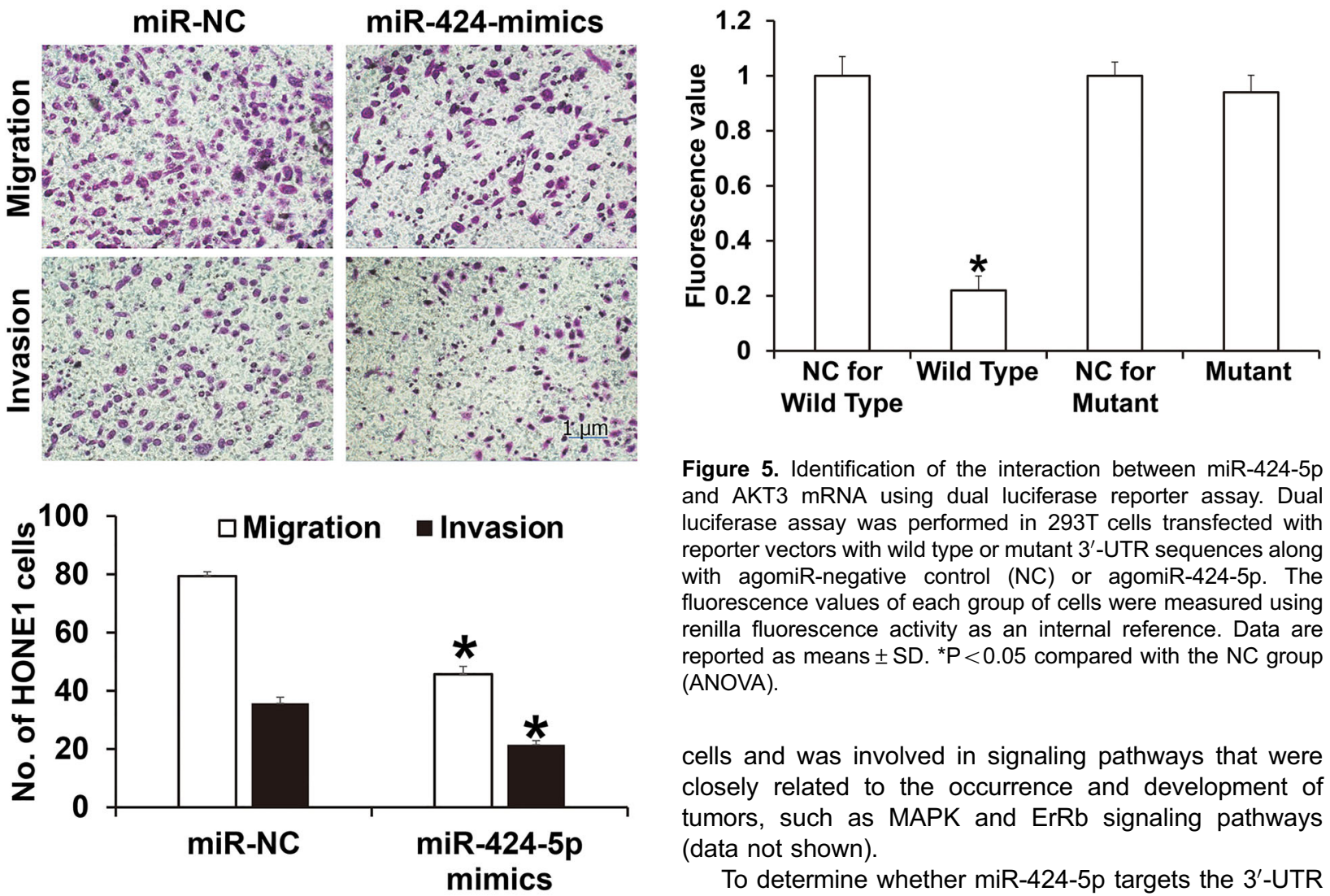

Figure 5. Identification of the interaction between miR-424-5p and AKT3 mRNA using dual luciferase reporter assay. Dual luciferase assay was performed in 293T cells transfected with reporter vectors with wild type or mutant $3^{\prime}$-UTR sequences along with agomiR-negative control (NC) or agomiR-424-5p. The fluorescence values of each group of cells were measured using renilla fluorescence activity as an internal reference. Data are reported as means $\pm S D$. ${ }^{*} \mathrm{P}<0.05$ compared with the $\mathrm{NC}$ group (ANOVA).

cells and was involved in signaling pathways that were closely related to the occurrence and development of tumors, such as MAPK and ErRb signaling pathways (data not shown).

To determine whether miR-424-5p targets the $3^{\prime}$-UTR of AKT3 mRNA, dual luciferase reporter assay was performed. The luciferase activity of cells co-transfected with agomiR-424-5p and the pMIR-REPORT-WT luciferase

Figure 4. Effect of miR-424-5p on the migration and invasion of nasopharyngeal carcinoma (NPC)-1 cells. Transwell assay was used to determine migration and invasion (scale bar: $1 \mu \mathrm{m}$ ). Data are reported as means $\pm S D$. ${ }^{*} P<0.05$ compared with the miR$\mathrm{NC}$ (negative control) group (t-test).

\section{Overexpression of $\mathrm{miR}-424-5 p$ inhibited migration and invasion of NPC-1 cells in vitro}

The number of NPC-1 cells that crossed the membrane in the miR-424-5p mimics group $(45.7 \pm 2.7)$ was significantly lower than that in the miR-NC group (79.4 \pm 1.5) $(P<0.05)$ (Figure 4). Invasion assay similarly showed that the number of NPC-1 cells that crossed the membrane in the miR-424-5p mimics group $(21.5 \pm 1.4)$ was also significantly lower than that in the miR-NC group $(35.7 \pm 2.1) \quad(P<0.05)$. These results suggested that overexpression of $\mathrm{miR}-424-5 \mathrm{p}$ inhibited the migration and invasion of NPC-1 cells in vitro.

\section{miR-424-5p bound the $3^{\prime}$-UTR seed region of AKT3 mRNA to regulate its expression}

The results identified 1507 potential target genes of miR-424-5p that were involved in MAPK, ErBb, and Ras signaling pathways (data not shown). Among all the potential target genes of miR-424-5p, AKT3 participated in the regulation of proliferation, apoptosis, and migration of reporter plasmids, containing the wild-type $3^{\prime}$-UTR of AKT3 mRNA, was significantly lower than that in the negative control group $(P<0.05)$. In contrast, the luciferase activity of cells co-transfected with agomiR-424-5p and the pMIR-REPORT-mutant luciferase reporter plasmid in which the binding sequence was mutated was not significantly different from that in the negative control group $(P>0.05)$ (Figure 5). These results suggested that miR424-5p bound the seed region in the $3^{\prime}-$ UTR of AKT3 mRNA to regulate its expression.

AKT3 rescued the inhibitory effect of miR-424-5p on the proliferation, migration, and invasion of NPC-1 cells

AKT3 expression in NPC-1 cells transfected with miR424-5p mimics was significantly lower than that in the miR-NC group $(P<0.05)$ (Figure $6 A)$. In addition, the level of AKT3 protein in NPC-1 cells transfected with both miR424-5p mimics and AKT3 was significantly higher than that in miR-NC group or miR-424-5p mimics $(P<0.05$ for both). CCK-8 assay showed that the proliferation of NPC1 cells transfected with miR-424-5p mimics was significantly reduced compared with that in the miR-NC group $(\mathrm{P}<0.05$ at all points), while the proliferation of NPC-1 

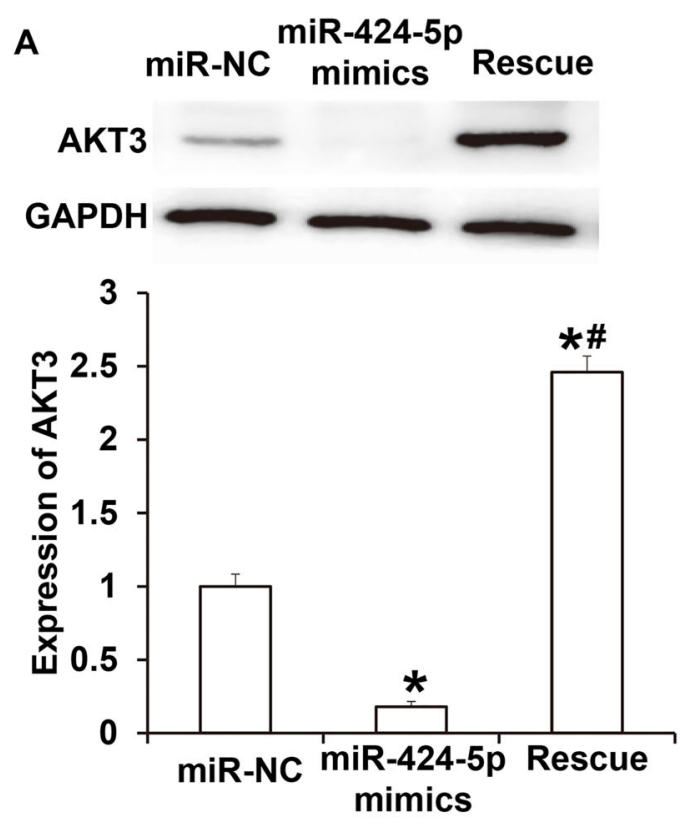

B

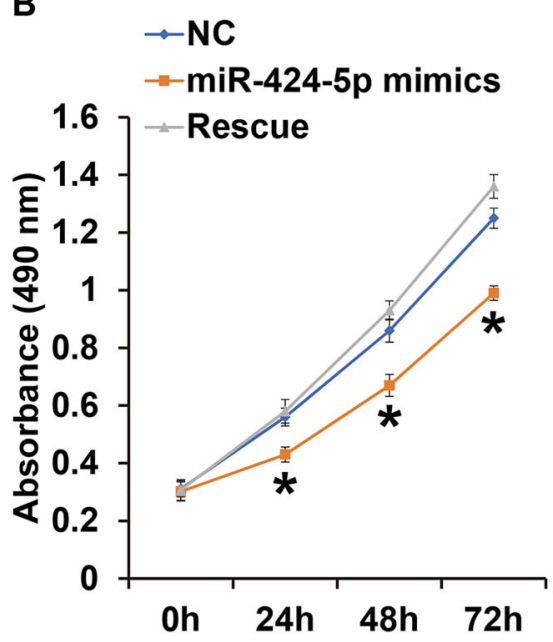

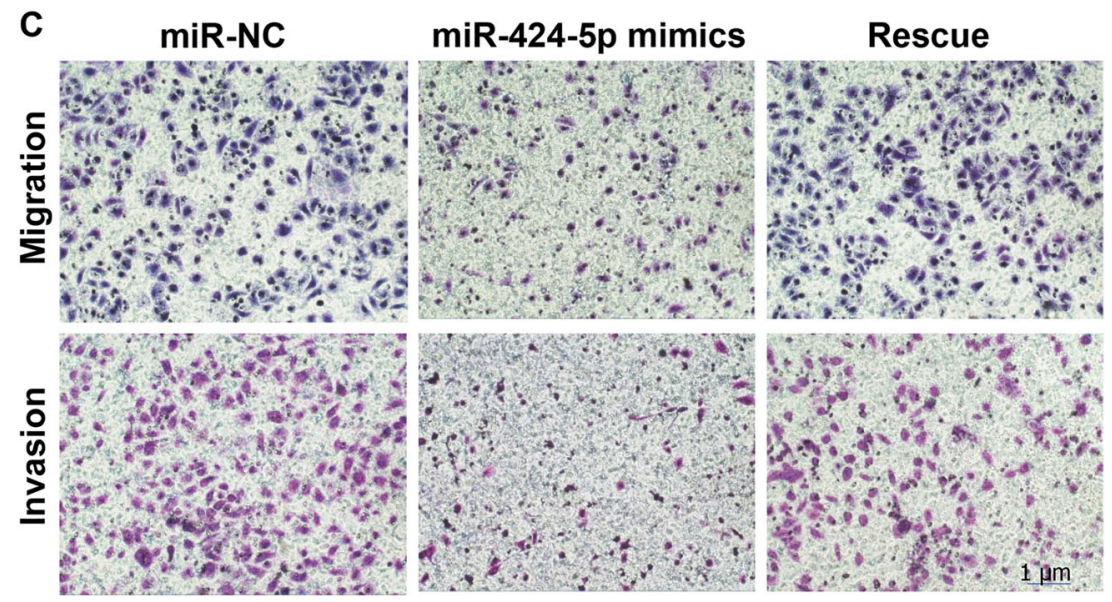

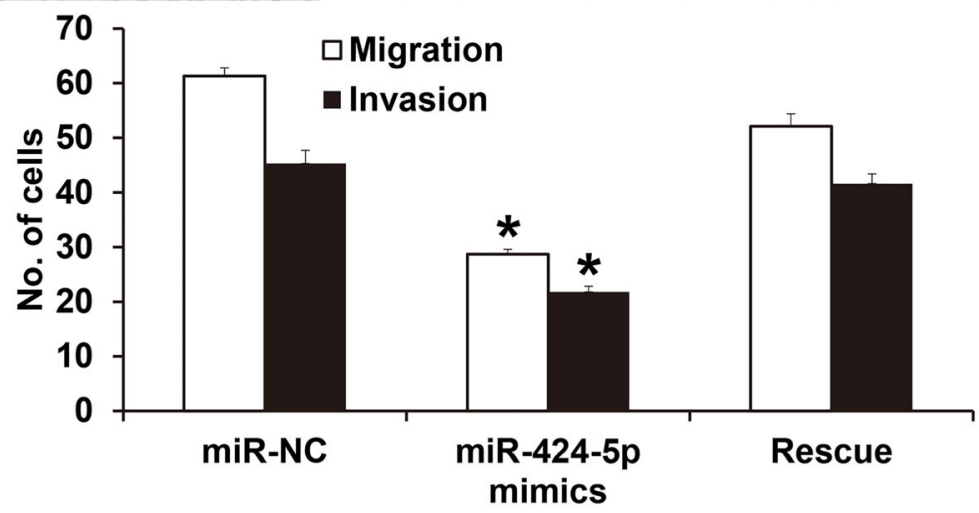

Figure 6. A, Expression of AKT3 cells in nasopharyngeal carcinoma (NPC)-1 cells transfected with miR-NC (negative control), miR-424$5 p$ mimics, or miR-424-5p mimics + AKT3 (Rescue). Western blotting was used to determine AKT3 protein expression. B, Proliferation of NPC-1 cells transfected with miR-NC, miR-424-5p mimics, or miR-424-5p mimics + AKT3 (Rescue). CCK-8 assay was used to detect cell proliferation. C, Migration and invasion of NPC-1 cells transfected with miR-NC, miR-424-5p mimics, or miR-424-5p mimics + AKT3 (Rescue). Scale bar: $1 \mu \mathrm{m}$. Transwell assay was used to evaluate migration and invasion. Data are reported as means $\pm S D$. * $P<0.05$ compared with the miR-NC group; ${ }^{\#} \mathrm{P}<0.05$ compared with the miR-424-5p mimics group ( $t$-test). 
cells transfected with both miR-424-5p mimics and AKT3 was similar to that in the miR-NC group $(P>0.05$ at all points) (Figure 6B). Transwell assay showed that while the migration and invasion of NPC-1 cells transfected with miR-424-5p mimics were significantly lower than those in the miR-NC group ( $\mathrm{P}<0.05$ for both), the migration and invasion of NPC-1 cells transfected with both miR-424-5p mimics and AKT3 were not different from those in miR-NC group ( $P>0.05$ for both) (Figure $6 \mathrm{C}$ ). These results suggested that AKT3 rescued the inhibitory effect of miR-424-5p on the proliferation, migration, and invasion of NPC-1 cells.

\section{Discussion}

NPC is the most common head and neck tumor in clinical settings and shows high malignancy. Cervical lymph node metastasis is prone to occur at the early stage of NPC. NPC tends to show resistance towards radiotherapy and chemotherapy, and the prognosis of NPC patients is poor (20). The recurrence and metastasis of NPC are the main contributing factors limiting its clinical treatment (21). Previous studies have shown that a large number of miRNA molecules are involved in the regulation of metastasis (22).

miR-424-5p is a newly discovered tumor-associated miRNA that is located at human Xq26.3. miR-424-5p is expressed by processing the $5^{\prime}$-terminus of has-miR- 424 (16). Several studies have shown that the expression and biological functions of miR-424-5p vary across different tumors. For example, the expression of miR-424-5p is reduced in liver cancer, gastric cancer, and osteosarcoma tissues, and miR-424-5p inhibits the proliferation and metastasis of these tumor cells (23). In contrast, expression of miR-424-5p is increased in squamous cell carcinoma of the esophagus, breast cancer, and pancreatic cancer, and it promotes the occurrence and development of these tumors (24). In the present study, we showed that miR-424-5p expression was significantly reduced in NPC tissues and negatively correlated with lymph node metastasis and TNM staging. Moreover, we isolated primary NPC cells with low expression of miR-425-5p, particularly NPC-1 cells. In vitro functional analyses showed that upregulation of miR424-5p expression inhibits the proliferation, migration, and invasion of NPC-1 cells, suggesting that miR-424-5p has oncogenic function and that reduced expression of miR424-5p in NPC may be related to the occurrence and

\section{References}

1. Wang F, Jiang C, Ye Z, Sun Q, Liu T, Xu M, et al. Efficacy and safety of nimotuzumab plus radiotherapy with or without cisplatin-based chemotherapy in an elderly patient subgroup (aged 60 and older) with nasopharyngeal carcinoma. Trans Oncol 2018; 11: 338-345, doi: 10.1016/j.tranon.2018.01. 013. development of NPC. The target genes of miR-424-5p are distinct in different tumors, and this may be the main reason for different the biological functions of miR-424-5p. For example, Zhou et al. reported that miR-424-5p regulates the Notch signaling pathway by targeting KDM5B and has tumor suppressor functions in cervical cancer (25). Wei et al. (18) showed that miR-424-5p regulates the TGF- $\beta$ signaling pathway by targeting Smad3 and promotes the proliferation of gastric cancer cells. Our bioinformatics results revealed the $A K T 3$ gene as a potential target of miR-424-5p. AKT is involved in the regulation of multiple downstream signaling pathways such as MAPK, Ras, and ErbB. Our qRT-PCR and western blotting data demonstrated that miR-424-5p downregulated the expression of AKT3 mRNA and protein. AKT is a key kinase in the PI3KJ AKT signaling pathway and has three subtypes, AKT1, AKT2, and AKT3 (26). The expression of AKT3 is elevated in a variety of tumors and is associated with abnormal proliferation, apoptosis resistance, and poor prognosis of the tumors (27). For example, AKT3 can make 40-60\% non-hereditary melanoma cells survive, and inhibition of AKT3 activity promotes the death of these cells (28). In addition, downregulation of AKT3 inhibits invasion and metastasis of glioma (29). The present study showed that elevation of AKT3 expression reversed the inhibitory effect of miR-424-5p on NPC cells and promoted the proliferation, migration, and invasion of NPC cells. Notably, the downstream signaling pathways of AKT3 and relevant molecules have not yet been identified. We plan on examining how AKT3 is related to the prognosis and clinical pathological characteristics of NPC and the correlation between AKT3 and $\mathrm{miR}-424-5 \mathrm{p}$ in clinical practice in future studies.

In conclusion, the present study demonstrated that miR-424-5p inhibited the proliferation, migration, and invasion of NPC cells by targeting AKT3 expression. The downregulation of miR-424-5p in NPC tissues was correlated with the occurrence and development of NPC.

\section{Acknowledgments}

The authors wish to thank their department and research team for their help and dedication. The study was supported by Science and Technology Project of Luzhou City (No. 2016-s-67). We thank Liwen Bianji, Edanz Editing China (www.liwenbianji.cn/ac), for editing the English text of a draft of this manuscript.
2. Chen $\mathrm{G}, \mathrm{Hao} \mathrm{H}, \mathrm{Ai} \mathrm{JW}$. Regulatory role of CDX2 and NOX4 expression associated with recurrent nasopharyngeal carcinoma. Eur Rev Med Pharmacol Sci 2018; 22: 450-455.

3. Lai W, Jia J, Yan B, Jiang Y, Shi Y, Chen L, et al. Baicalin hydrate inhibits cancer progression in nasopharyngeal carcinoma by affecting genome instability and splicing. 
Oncotarget 2018; 9: 901-914, doi: 10.18632/oncotarget. 22868.

4. Jin T, Jiang F, Jin QF, Piao YF, Chen XZ. Endostar combined with gemcitabine and cisplatin chemotherapy for patients with metastatic nasopharyngeal carcinoma: an update. Trans/ Oncol 2018; 11: 286-291, doi: 10.1016/j.tranon.2018. 01.002.

5. Li Y, Huang X, Jiang J, Hu W, Hu J, Cai J, et al. Clinical variables for prediction of the therapeutic effects of bevacizumab monotherapy in nasopharyngeal carcinoma patients with radiation-induced brain necrosis. Int $J$ Rad Oncol Biol Phys 2018; 100: 621-629, doi: 10.1016/j.jirobp. 2017.11.023.

6. Xu S, Li Y, Lu Y, Huang J, Ren J, Zhang S, et al. LZTS2 inhibits $\mathrm{PI} / 3 \mathrm{~K} / \mathrm{AKT}$ activation and radioresistance in nasopharyngeal carcinoma by interacting with p85. Cancer Lett 2018; 420: 38-48, doi: 10.1016/j.canlet.2018.01.067.

7. Jin YN, Zhang WJ, Cai XY, Li MS, Lawrence WR, Wang SY, et al. The characteristics and survival outcomes in patients aged 70 years and older with nasopharyngeal carcinoma in the intensity-modulated radiotherapy era. Cancer Res Treat 2019; 51: 34-42, doi: 10.4143/crt.2017.551.

8. Lee HF, Lan JH, Chao PJ, Ting HM, Chen HC, Hsu HC, et al. Radiation-induced secondary malignancies for nasopharyngeal carcinoma: a pilot study of patients treated via IMRT or VMAT. Cancer Manag Res 2018; 10: 131-141, doi: 10.2147/CMAR.S145713.

9. Ye P, Shi Y, An N, Zhou Q, Guo J, Long X. miR-145 overexpression triggers alteration of the whole transcriptome and inhibits breast cancer development. Biomed Pharmacother 2018; 100: 72-82, doi: 10.1016/j.biopha. 2018.01.167.

10. Zhou YL, Yang QQ, Yan YY, Zhu C, Zhang L, Tang JB. Localized delivery of miRNAs targets cyclooxygenases and reduces flexor tendon adhesions. Acta Biomater 2018; 70: 237-248, doi: 10.1016/j.actbio.2018.01.047.

11. Li Z, Zhang W, Huang Y. MiRNA-133a is involved in the regulation of postmenopausal osteoporosis through promoting osteoclast differentiation. Acta Biochim Biophys Sin 2018; 50: 273-280, doi: 10.1093/abbs/gmy006.

12. Wu WL, Wang WY, Yao WQ, Li GD. Suppressive effects of microRNA-16 on the proliferation, invasion and metastasis of hepatocellular carcinoma cells. Int $\mathrm{J} \mathrm{Mol} \mathrm{Med} \mathrm{2015;} \mathrm{36:}$ 1713-1719, doi: 10.3892/ijmm.2015.2379.

13. Li BS, Zuo QF, Zhao YL, Xiao B, Zhuang Y, Mao XH, et al. MicroRNA-25 promotes gastric cancer migration, invasion and proliferation by directly targeting transducer of ERBB2, 1 and correlates with poor survival. Oncogene 2015; 34: 2556-2565, doi: 10.1038/onc.2014.214.

14. Zhang L, Guo X, Zhang L, Yang F, Qin L, Zhang D, et al. SLC34A2 regulates miR-25-Gsk3beta signaling pathway to affect tumor progression in gastric cancer stem cell-like cells. Mol Carcinog 2018; 57: 440-450, doi: 10.1002/mc.22 768.

15. He J, Qi H, Chen F, Cao C. MicroRNA-25 contributes to cisplatin resistance in gastric cancer cells by inhibiting forkhead box O3a. Oncol Lett 2017; 14: 6097-6102, doi: 10. 3892/ol.2017.6982.

16. Connolly M, Paul R, Farre-Garros R, Natanek SA, Bloch S, Lee $J$, et al. miR-424-5p reduces ribosomal RNA and protein synthesis in muscle wasting. $J$ Cachexia Sarcopenia Muscle 2018; 9: 400-416, doi: 10.1002/jcsm.12266.

17. Liu J, Gu Z, Tang Y, Hao J, Zhang C, Yang X. Tumoursuppressive microRNA-424-5p directly targets CCNE1 as potential prognostic markers in epithelial ovarian cancer. Cell Cycle 2018; 17: 309-318, doi: 10.1080/15384101.2017. 1407894.

18. Wei S, Li Q, Li Z, Wang L, Zhang L, Xu Z. Correction: miR424-5p promotes proliferation of gastric cancer by targeting Smad3 through TGF-beta signaling pathway. Oncotarget 2017; 8: 34018, doi: 10.18632/oncotarget.12092.

19. Livak KJ, Schmittgen TD. Analysis of relative gene expression data using real-time quantitative PCR and the 2(-Delta Delta C(T)) Method. Methods 2001; 25: 402-408, doi: 10. 1006/meth.2001.1262.

20. Du M, Huang T, Wu J, Gu JJ, Zhang N, Ding K, et al. Long non-coding RNA n326322 promotes the proliferation and invasion in nasopharyngeal carcinoma. Oncotarget 2017; 9: 1843-1851, doi: 10.18632/oncotarget.22828.

21. Ng WT, Ngan RKC, Kwong DLW, Tung SY, Yuen KT, Kam MKM, et al. Prospective, multicenter, phase 2 trial of induction chemotherapy followed by bio-chemoradiotherapy for locally advanced recurrent nasopharyngeal carcinoma. Int $J$ Radiat Oncol Biol Phys 2018; 100: 630-638, doi: 10.1016/ j.jijrobp.2017.11.038.

22. Wang $\mathrm{Y}$, Zhao $\mathrm{Q}$, Lan N, Wang S. Identification of methylated genes and miRNA signatures in nasopharyngeal carcinoma by bioinformatics analysis. Mol Med Rep 2018; 17: 4909-4916, doi: 10.3892/mmr.2018.8487.

23. Wang F, Wang J, Yang X, Chen D, Wang L. MiR-424-5p participates in esophageal squamous cell carcinoma invasion and metastasis via SMAD7 pathway mediated EMT. Diagn Pathol 2016; 11: 88, doi: 10.1186/s13000-016-0536-9.

24. Zhang M, Zeng J, Zhao Z, Liu Z. Loss of MiR-424-3p, not miR-424-5p, confers chemoresistance through targeting YAP1 in non-small cell lung cancer. Mol Carcinog 2017; 56: 821-832, doi: 10.1002/mc.22536.

25. Zhou Y, An Q, Guo RX, Qiao YH, Li LX, Zhang XY, et al. miR424-5p functions as an anti-oncogene in cervical cancer cell growth by targeting KDM5B via the Notch signaling pathway. Life Sci 2017; 171: 9-15, doi: 10.1016/j.lfs.2017. 01.006 .

26. Fu JH, Yang S, Nan CJ, Zhou CC, Lu DQ, Li S, et al. MiR182 affects renal cancer cell proliferation, apoptosis, and invasion by regulating PI3K/AKT/mTOR signaling pathway. Eur Rev Med Pharmacol Sci 2018; 22: 351-357.

27. Yeganeh PN, Richardson C, Bahrani-Mostafavi Z, Tait DL, Mostafavi MT. Dysregulation of AKT3 along with a small panel of mRNAs stratifies high-grade serous ovarian cancer from both normal epithelia and benign tumor tissues. Genes Cancer 2017; 8: 784-798, doi: 10.18632/genesandcancer. 164.

28. Madhunapantula SV, Robertson GP. Targeting protein kinase-b3 (akt3) signaling in melanoma. Expert Opin Ther Targets 2017; 21: 273-290, doi: 10.1080/14728222.2017. 1279147.

29. Joy A, Kapoor M, Georges J, Butler L, Chang Y, Li C, et al. The role of AKT isoforms in glioblastoma: AKT3 delays tumor progression. J Neuro Oncol 2016; 130: 43-52, doi: 10.1007/s11060-016-2220-z. 